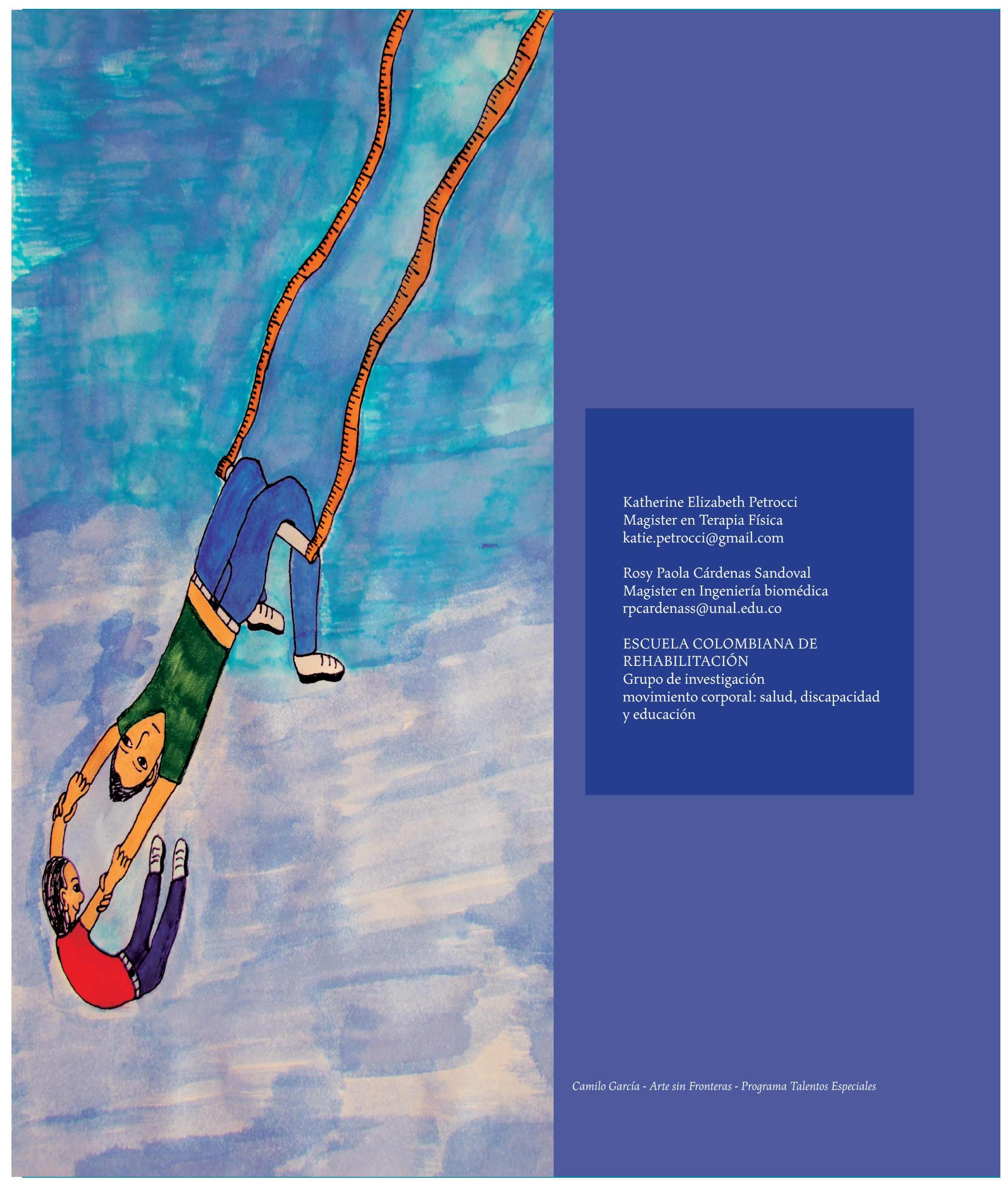




\title{
LA MEDICIÓN DEL CONTROL POSTURAL CON ESTABILOMETRÍA- UNA REVISIÓN DOCUMENTAL
}

\section{Measurment of Postural Control Through Stabilometry- a Literature Review}

Fecha de Recepción: 5 de Junio de 2011 - Fecha de Aprobación: 15 de Agosto de 2011

\begin{abstract}
RESUMEN
La estabilometría es el estudio del equilibrio que permite analizar el control postural y su relacion con la estabilidad en bípedo. Herramientas de alta tecnología se utilizan para medir la estabilidad en bípedo de forma cuantitativa con base en la posición del centro de gravedad de la persona determinado por la distribución de presiones plantares. Este artículo revisa el conocimiento científico sobre los conceptos en el trastorno de la estabilometría y la variedad de uso de estas herramientas en diferentes poblaciones y diagnósticos con base en artículos científicos encontrados en los últimos diez años en revistas indexadas. Se recomienda expandir el uso de las plataformas de fuerza que existen en Colombia a la estabilometría.
\end{abstract}

PALABRAS CLAVE

Estabilometría, plataforma de fuerza, equilibrio, estabilidad postural

\section{ABSTRACT}

Stabilometry is the study of balance which permits the analysis of postural control as it relates to stability in standing. High tech tools are used to measure standing stability cuantitatively based on the position of the center of gravity of the person determined by his/ her distrubution of plantar pressures. This article reviews scientific knowlege regarding the concepts surrounding stabilomitry and the wide variety of uses of these tools in different populations and diagnoses based on scientific articles found in the last ten years in indexed journals. Expanding the use of the force plates that exist in Colombia to include stabilometry is recommended.

KEY WORDS

Stabilometry, force plate, balance, postural stability 


\section{INTRODUCCIÓN}

$\mathrm{E}$ análisis de los trastornos en la postura y el equilibrio de los individuos, hoy en día, incluye el uso de una gran variedad de métodos entre ellos: el análisis cualitativo, cuyos resultados son descriptores de la postura; herramientas cuantitativas, tales como escalas, conocidas como la Prueba de Romberg (1), la Escala de Berg (2), Star Excursion Balance Test (SEBT) (3), cuyos resultados cuantitativos se basan en el análisis subjetivo de varias actividades; $y$ herramientas de alta tecnología, cuyos resultados son valores precisos que representan el desplazamiento de la proyección del centro de gravedad (CG) corporal sobre una superficie. Esta última se conoce como estabilometría, la cuál hace referencia al estudio del equilibrio / balance corporal humano.

La habilidad en humanos de mantener en equilibrio el centro de masa corporal (CM) contra las fuerzas de la gravedad se conoce como el equilibrio postural y se da como resultado del control postural, una interacción entre el individuo, el entorno y la actividad. El desarrollo del control postural requiere del desarrollo individual y de la integración entre el sistema sensorial (vestibular, visión, somatosensorial), el sistema motor y el control inconsciente cerebeloso. La ejecución del control postural se basa en la organización cerebral de las conexiones sesorial-motoras y las subsecuentes sinergias musculares que forman las estrategias utilizadas para mantener el equilibrio. Estas interacciones, conexiones y estrategias están moduladas con base en cambios que ocurren en el nivel celular en cualquiera de los sistemas integrantes y que están representadas en pruebas clínicas. En el sistema músculo-esquelético por ejemplo, cambios en la organización fibral de los tejidos puede demostrarse en cambios notados en la masa corporal, fuerza y resistencia muscular o rangos de movilidad. Estos cambios que son parte del desarrollo neuromuscular en infantes, causan las constantes modificaciones durante la madurez física hasta la vejez (4).

La valoración postural de cada uno de los sistemas integrantes se puede realizar a partir de la medición del manejo del CG corporal, su relación con los límites de la estabilidad y la alineación corporal. Los límites de estabilidad están formados por la base de sustentación que en la posición bípeda es el área de longitud anterior posterior y el ancho medio lateral de la huella plantar. El centro de presión (CP) o baricentro corporal, es la representación bi-dimensional del punto en el cual se proyecta el vector de reacción del suelo, representa el promedio de todas las presiones sobre la superficie del área en contacto en el plano sagital (desviaciones en sentido antero-posterior) y en el plano coronal (desviaciones en sentido latero-lateral) $(5,6)$. La estabilometría registra de forma cuantitativa, a través de una plataforma de sensores de presión, las desviaciones en el CP. Se reconoce que entre más alineado el cuerpo, menos gasto de energía requiere para mantenerse en equilibrio y que entre menores las oscilaciones del CP mejor es la ejecución del control postural (6).

El control postural se ejecuta en posiciones estáticas, cinéticas y dinámicas y la valoración del equilibrio postural en estas tres situaciones se conoce como equilibrio estático, reactivo y dinámico (4). El equilibrio estático es cuando el individuo se encuentra sometido únicamente a la acción de la gravedad y el cuerpo se encuentra dentro de la base de sustentación. El equilibrio reactivo es el que se activa cuando alguna fuerza perturba la posición y ocasiona un desplazamiento del centro de gravedad (4). Las reacciones equilibratorias ocurren ante perturbaciones en la superficie de apoyo o desplazamientos de segmentos del cuerpo fuera de la base de sustentación con el fin de recuperar el equilibrio perdido (7). Y por último, el equilibrio dinámico actúa cuando el individuo realiza un movimiento parcial o total de su cuerpo fuera de la base de sustentación, también se conoce como equilibrio anticipatorio $(4,8,9)$. La estabiliometría permite el análisis de las tres clasificaciones del equilibrio, a través de herramientas tecnológicas, las cuales utilizan plataformas de fuerza para medir el centro de presión plantar.

Estas plataformas de sensores de presión se encuentran disponibles en Colombia en laboratorios de análisis computarizado del movimiento y son utilizadas para el análisis biomecánico de la marcha en ortopedia y neurología. Sin embargo, se encuentran subutilizadas ya que se desconocen los valores de normalidad y sus usos potenciales en el diagnóstico y tratamiento de trastornos del equilibrio y en la presencia de patología. Los autores de un estudio desarrollado a la Universidad del Cauca, Colombia, reconocen la necesidad de validar el estabilómetro y protocolo en diferentes poblaciones (10).

Por lo tanto, la presente revisión documental tiene como objetivo identificar cuál es el conocimiento científico de la herramienta tecnológica de estabilometría en los últimos diez años con el fin de expandir el uso de las plataformas de fuerza que existen en Colombia. Específicamente, se busca evaluar el uso general de la herramienta tecnológica de estabilometría según su aplicación práctica, evaluar las conclusiones de los artículos de investigación según la población de uso y definir recomendaciones sobre el uso general de la herramienta tecnológica de estabilometría en Colombia.

\section{METodología}

Se realizó una búsqueda con palabras claves (stabilometry, posturography, force plate, vestibular, balance, equilibrium, postural stability, baropodometric) entre los años 2001 y 2011 en bases de datos de revistas indexadas tales como PubMed, Cochrane y HinariHealth Internetwork.

\section{RESULTADOS}

Se encontraron 36 artículos científicos a través de la búsqueda mencionado anteriormente y 4 artículos adicionales de los años 1982 a 2000 que se encontraron referenciados por los autores de los primeros artículos y que se consideran pertinentes para los propósitos de la presente revisión documental.

Los resultados están organizados de la siguiente manera: principios de la estabilometría, herramientas de alta tecnología en la estabilometría, usos de la herramienta de alta tecnología en la investigación de la estabilometría.

\section{Principios de la estabilometría}

Se realiza la estabilometría sobre un estabilómetro o baropodómetro, utilizando una plataforma de fuerza que provee las coordenadas del CP en el plano sagital y coronal según las oscilaciones del 
cuerpo. El CP es diferente al centro de masa (CM) que es el punto en el espacio tridimensional, representa el promedio de la masa o peso corporal y está ubicado dentro del cuerpo. El CG es el punto que representa la CM teniendo en cuenta la fuerza de la gravedad. La superficie plantar del pie funciona como la interación entre el cuerpo y el superficie externa durante la dinámica postural (11). El $\mathrm{CP}$ es el punto resultante del intercambio de las fuerzas del pie y del piso. En otras palabras, es la fuerza de reacción del piso aplicada a cada punto que mantiene contacto entre la superficie plantar del pie con la superficie de la base y está ubicado en la superficie plantar del pie en una actividad unipodal y entre los dos pies en una actividad bipodal. Su cálculo depende del conocimiento de otras variables de orden cinético, como las fuerzas de reacción y los momentos. La fuerza de reacción en cada pie es igual y opuesta a la masa corporal, su unidad se expresa en [m], y los momentos, equivalen a la relación entre los vectores de fuerza y la distancia hacia el centro articular, su unidad se define en $[\mathrm{Kg} \mathrm{m}]$. Estos momentos pueden ser clasificados en: la fuerza de reacción, el peso e inercia (12).

\section{Herramientas de alta tencnología en la estabilometría}

Las herramientas de alta tecnología utilizadas en la estabilometría requieren de una plataforma de fuerza y de un software que permite el análisis de los datos. Dichas herramientas permiten la medición del equilibrio estático, reactivo y dinámico, incluyen dos tipos, la plataforma de fuerza dinámica, la cual puede crean perturbaciones externas y la plataforma de fuerza estática, cuyos componentes se encuentran fijos dentro del laboratorio de análisis de marcha y no permite producir perturbaciones externas.

Las plataformas de fuerza estáticas se diferencian entre sí en términos del tamaño de la superficie, el número de los sensores, la
BJL Group (14), Biodex Medical Systems Inc (15), AMTI Advanced Medical Technology Inc (16), Physical Support (17), Anima Corporation (18), Kistler (19), Diasu Company (20), Guy Capron (21).

Otra clase de herramienta de alta tecnología en la estabiliometría es la plataforma de fuerza dinámica la cual simula perturbaciones externas. Uno de estos sistemas es de la empresa Neurocom International y SMS Healthcare $(15,22,23)$. Algunas de las perturbaciones externas que pueden mencionarse se aprecian en la Figura 1 (24).

Los parámetros del balance que pueden ser controlados por el experimentador y que pueden afectar el control postural son: 1) Pico de aceleración, 2) Tiempo pico de aceleración, 3) Pico de velocidad y 4) Amplitud de los desplazamientos de la superficie de soporte.

Los elementos del control postural que pueden ser manipulados son: 1) El tamaño de la base de soporte, el cual puede ser reducido para ejecutar tareas de balance cambiantes. 2) La retroalimentación visual, la cual puede ser disminuida. Por ejemplo, cerrar los ojos. 3) La retroalimentación propioceptiva. Por ejemplo, superficie de soporte inestable. 4) Las aferencias sensoriales, mediante la utilización de perturbaciones con el fin de manipular selectivamente uno o más elementos específicos del control postural. Así se puede obtener un mejor entendimiento de los principios mecánicos, fisiológicos y patofisiológicos. Ejemplos incluyen movimiento de la escena visual, estimulación vestibular galvánica o vibración en el tendón. 5) La "gravicepción" como un cuarto posible origen de información aferente para el control postural. Ejemplo de ellos es la realización de pruebas bajo el agua. 6) La carga cognitiva, cambios en este factor permiten incrementar la complejidad de la prueba. Por ejemplo, solicitar la ejecución de tareas secundarias o tareas repetitivas mientras los sujetos mantienen el balance (24).

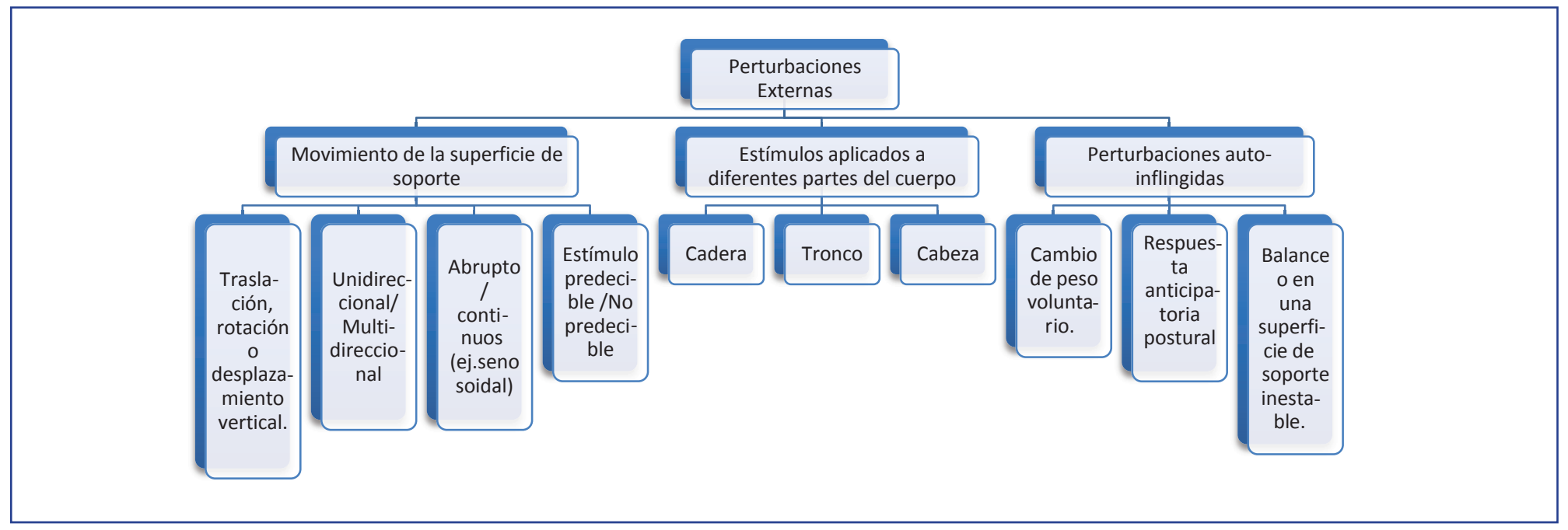

Figura 1: Tipos de perturbaciones externas que se simulan en la plataforma de fuerza dinámica.

frecuencia de toma de datos de los sensores y el software que acompaña a la plataforma lo cual permite una variedad en los cálculos y gráficos. Se encuentran herramientas de alta tecnología de estabilometría de los siguientes fabricantes, mencionados en los artículos referenciados en esta revisión: Tekscan (13), MIDI-CAPTEURS S.A.
Adicionalmente a la plataforma, la estabilometría requiere de un software que es responsable por el análisis y display de los datos. El primer software de estabilometría o baropodometría para medir la presión plantar en una plataforma dual fue desarrollado por Piero Galasso en 1987 en conjunto con la Facultad de Rehabilitación de la 


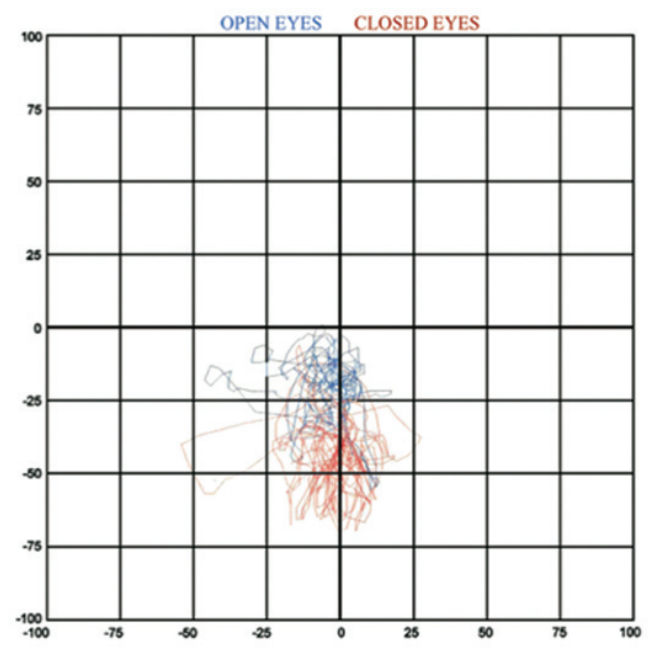

Figura 2: Estatokinesiogramo ऍ̌

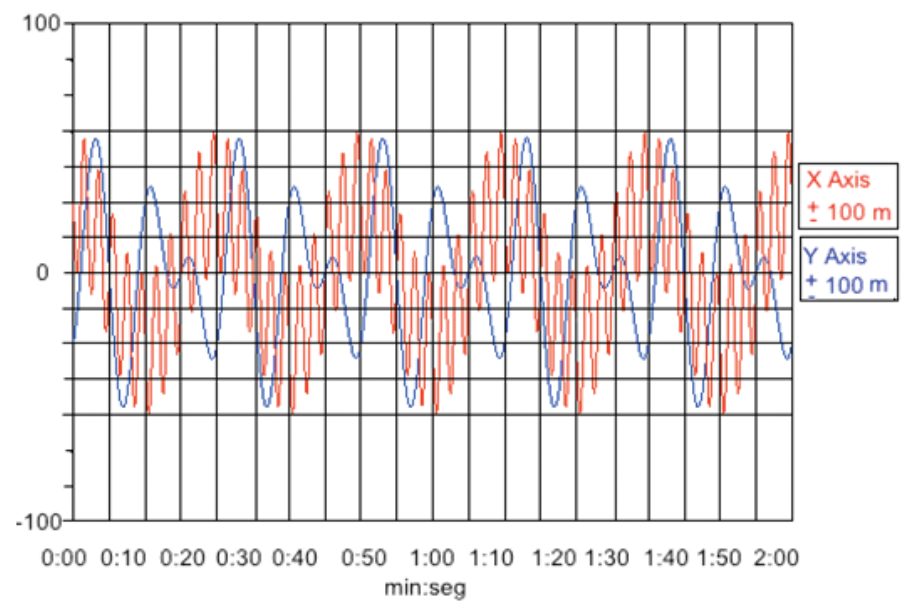

Figura 3: Estabilogramo. Ť Imagen simulada en el software Scilab.

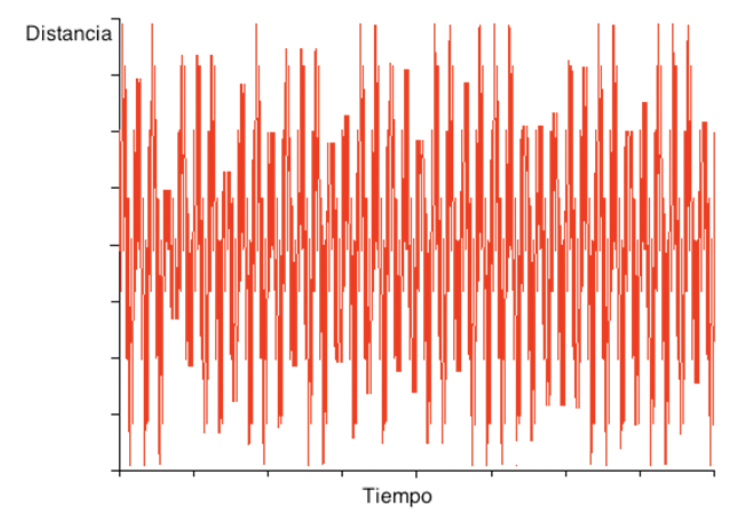

Figura 4: Velocidad de las Oscilaciones. $\mp$ Imagen simulada en el software Scilab. ${ }^{*}(24) \check{\mathrm{T}}(20) \mp(6)$
Universidad de Montpellier (25). Internamente, funciona como un conjunto de instrucciones escritas en un lenguaje de programación específico como matlab para calcular la variable de interés, en este caso, el centro de presión plantar. El cálculo numérico depende de otras variables de entrada como la fuerza de reacción y el peso corporal, cuyas magnitudes se obtienen digitamente por medio de un conversor de señal analógica a digital conectado a los sensores de presión de la plataforma de fuerza. La interfaz gráfica del software permite al usuario del estabilómetro visualizar los datos obtenidos en el tiempo (26).

Estos datos tomados sobre un periodo de tiempo se muestran en varias formas de gráficos que han sido estandarizadas por la Sociedad Internacional de Posturografía y reportados por Kapteyn et al 1983 (6). El estatokinesiogramo (Figura 2) (20) muestra los movimientos del $\mathrm{CP}$ o las oscilaciones corporales durante un periodo de tiempo. El estabilogramo (Figura 3) (20) muestra los desplazamientos del CP anterior-posterior en plano sagital sobre el eje Y y latero-lateral en el plano coronal sobre el eje X (20). Otro gráfico es el de velocidad que permite visualizar la velocidad instantánea del $\mathrm{CP}$ que corresponde a las oscilaciones del $\mathrm{CP}$ en los dos planos sobre el tiempo (Figura 4) (6). El último gráfico utilizado es el de radar balance (6) que generaliza en una forma específica la dirección de las oscilaciones principales. Similar a este último es el elíptico de confianza que es un óvalo que contiene un $95 \%$ de probabilidad las oscilaciones corporales del CP.

Con tanta variedad en las funciones y especificaciones técnicas de las herramientas de alta tecnología de la estabilometría, el Instituto Nacional de Salud de Italia (ISS) identificó los siguientes componentes importantes para valorar: la falta de precisión que viene de diferencias en la tecnología de los sensores, la resolución espacial de la matriz, el rango de la presión, calibración, y el procesamiento de los datos para después estandarizar el significado de los resultados obtenidos sobre los datos (27).

Es importante tener en mente que estas herramientas no incluyen los sistemas compuestos por cintas delgadas, como el de Tactile Pressure Indicating Sensor Film PressureX by Sensor Products Inc., cuya función es hacer un análisis cualitativo depictado en una imagen topográfica de baja resolución de la distribución máxima de la presión. Estos sistemas son para uso y deshecho y no deberían ser utilizado en un análisis cuantitativo de la Presión (27).

La precisión de la medición del CP es uno de los temas identificado por la ISS como importante para ser cuantificado por las herramientas. El estudio de Middleton et al. (28), tenía como objetivo evaluar la exactitud en la determinación de centro de presión con una plataforma de fuerza utilizando un modelo de un solo punto y un modelo de "dos pies". En la prueba con el modelo de un solo punto se encontró que la precisión del $\mathrm{CP}$ era mayor entre más cerca se encontrara a la línea media de la plataforma y que disminuía al alejarse de la misma. En el modelo "dos pies" se utilizaron dos formas hechas de hierro con una fuerza vertical de $300 \mathrm{~N}$ que representaron a los pies humanos. Se determinó que los errores en la ubicación del $\mathrm{CP}$ en bípedo son menores a $2 \mathrm{~mm}$ con los pies posicionados simétricamente alrededor del centro de la plataforma de fuerza (28). 
Se han utilizado las herramientas de alta tecnología de la estabilometría como instrumento en estudios científicos en distintas poblaciones: sana $(7,29,30,31,32,33)$, adultos mayores $(34,35)$, bariátrica $(36,37)$, deportistas $(17,38,39)$, personas con alteraciones neurológicas: enfermedad cerebro vascular (ECV) $(15,40)$, enfermedad de parkinson $(18,41)$, ataxia cerebelosa $(42)$, resección de tumor $(20)$, mielopatía cervical (43), vértigo BPPV (14), migraña y dolor de cabeza $(44,45)$, desorden vestibular (46), extropía (47) y estrabismo (48).

El estabilómetro ha sido utizado como instrumento independiente y en conjunto de instrumentos electromiográficos y cinemáti$\cos$, mediante el uso de electrodos de superficie, aguja y de alambre insertados, y sensores de movimiento con análisis óptico, respectivamente.

Dentro de los estudios sobre la población sana se estudiaron los valores de normalidad (valores de la simetría de las extremidades entre lados corporales en bípedo, la relación entre las variables estabilométricas, la velocidad promedio y la amplitud de movimientos del $\mathrm{CP}$ ) sobre los factores de edad, sexo, altura, peso, nivel de actividad. Los resultados no son contundentes por ejemplo, un estudio reportó que no habían diferencias significativas en el patrón de oscilación en bípedo entre la altura, edad (rango 20-54 años), peso (49) mientras que otros, los estudios por Ekdahl et al. Kollegger et al. y Hageman et al. citados en el estudio por Nordahi et al. (49), muestran que oscilaciones corporales aumentan con la edad (29) y que hombres muestran oscilaciones mayores que mujeres en la condición de prueba con los ojos abiertos (49). Adicionalmente, Ageberg et al. (29), mostraron aumentos en la oscilación de $10 \%$ por cada 10 años de edad y por tanto recomiendan parejar lo sujetos por edad y sexo en la realización de investigaciones con un grupo control de sujetos sanos. El estudio por Brunner et al. (11) muestra las variaciones en la forma del pie y su relación con la distribución de descarga y el patrón del arco plantar con respecto al apoyo completo del pie, y otro estudio muestra las variaciones en la forma del pie con respecto al peso corporal en sujetos sanos (32). En adición, estudios con la población sana muestran "efectos del aprendizaje", se presentan mejores resultados en la prueba cuanto mayores repeticiones se realizan de la misma y cuanto menor sea el intervalo de tiempo entre las pruebas. (49)

Se estudiaron los efectos de tratamientos e intervenciones utilizando estabilometría como el instrumento de medición. Unos de estos estudios $(34,35)$ miraron los efectos en la estabilometría de programas de fisioterapia con énfasis en estimulación propioceptiva, multi-sensorial comparado con programas de fisioterapia estándares o con enfoque en fortalecimiento muscular en adultos mayores $(34,35)$. Estos programas mostraron mayor reclutamiento del tibial anterior y gastrocnemio durante la estabilometría, disminución de las oscilaciones corporales $(34,35)$, y mejor desempeño en la prueba Get up and Go (Levantarse y Avanzar) (35). El artículo de Boza (40), estudió la correlación entre el equilibrio, la marcha y la discapacidad en ECV y mostraron los valores de la superficie de apoyo plantar del pie pléjico que se correlacionan con valores de la medida de independencia funcional, Functional Independence Measure (FIM), la velocidad de la marcha y el equilibrio. Adicionalmente, los autores mostraron que los sujetos que usaron órtesis antiequino presentaron mayor asimetría en el equilibrio que los sujetos que no usaron ortesis. Otros estudios estudiaron los efectos de estiramiento de los grupos musculares en los miembros inferiores sobre el equilibrio dinámico en mujeres activas (38), los efectos de manipulación articular del cuello del pie sobre equilibrio esático en atletas de hockey (39).

La estabilometría ha sido utilizada para medir el balance unipodal y la prueba de salto unipodal a distancia en el diagnóstico de patologías incluyendo la función en los ligamentos de la rodilla (29). Adicionalmente, ha sido utilizado en el diagnóstico de deficiencias en la estabilidad postural como fue estudiado en el artículo por $\mathrm{Ge}-$ rosa (20). Los autores estudiaron los efectos de la estabilometría a 6, 12, 18 meses después de la radiocirugía Gamma Knife, en pacientes con resección de tumores. Los resultados mostraron una disminución en la anormalidad de equilibrio en el sentido anterior-posterior y latero-lateral de $62 \%$ a $32 \%$ y por lo tanto los autores recomendaron utilizar la estabilometría como una herramienta de medición en el seguimiento de resección de tumores de este tipo. Otro estudio, Rossi (44), tenía como objetivo determinar la prevalencia de alteraciones en el equilibrio en pacientes quienes presentaron dolores de cabeza, migraña y dolor de cabeza tipo tensional. Los autores encontraron una asociación entre la alteración en el equilibrio y el uso significativo de los músculos craneocervicales posteriores en el grupo de sujetos con dolor de cabeza tipo tensión. Adicionalmente, encontraron que el grupo de sujetos con migraña presentaron alteraciones en el equilibrio de origen visual (44).

La estabilometría ha sido utilizado en estudios para determinar la validez de otras escalas o componentes de escalas que tratan de la medición subjetivo del equilibrio. Uno de estos estudios, Ganesan 2010 (41), miró la correlación del ítem 30 ("Pull Test") de la escala Unified Parkinson Diseases Rating Scale (UPDRS) que pretende medir los grados de estabilidad postural en personas con enfermedad de Parkinson (EP). Ellos mostraron que sujetos con EP que no presentaron alteraciones según el ítem 30 de la prueba clínica presentaron valores menores a los límites de estabilidad en la dirección diagonal hacia delante derecha y hacia atrás izquierda, lo cual puede indicar la presencia de inestabilidad postural antes de lo predicho por la escala UPDRS y muestra la invalidez de la prueba ya que solamente mide alteraciones antero-posteriores (41).

El estabilómetro ha sido utilizado como herramienta de entrenamiento para determinar los efectos de retroalimentación visual y auditiva sobre el equilibrio en bipedestación en usuarios con accidente cerebrovascular. Este estudio por Barclay et al 2008 (15), mostró mejorías significativas en la estabilometría en sujetos participantes en entrenamiento que utilizaron retroalimentación visual y retroalimentación visual y auditiva concomitantes. A la vez, este estudio mostró que los mismos sujetos no mostraron mejoramientos en las pruebas clínicas de equilibrio Berg Balance Scale y prueba de levantarse y avanzar (15).

\section{DISCUSIÓN}

La literatura científica muestra la razón por la cual la estabilometría es necesaria y superior en algunos sentidos que las pruebas clínicas, esto se debe a la capacidad para medir cuantitativamente 
las variables asociadas a la estabilidad postural y así, ofrecer validez a las pruebas clínicas.

Las herramientas de alta tecnología de estabilometría se encuentran disponibles en Colombia en Laboratorios para Análsis Computarizado del Movimiento. Estas herramientas son utilizadas con mayor frecuencía en el área de la ortopedia para el análisis de las presiones plantares dinámicas durante la marcha, sin embargo se encuentran subutilizados en el área de la estabilometría ya que según lo reportado por la literatura aún no se evidencian aplicaciones sobre estabilidad postural en Colombia.

Principalmente, se recomienda el uso de la estabilometría para estudiar los valores de normalidad de la población colombiana sobre el control postural en circunstancias estáticas. Se puede organizar este conocimiento, sobre el rango y promedio de los valores, por edad según la etapa de la vida (niñez, adoloscencia, adulto, adulto mayor), sexo, o población específica (trabajadores según la actividad funcional, deportistas según el deporte) (50). Adicionalmente, al conocer estos valores sobre ambos miembros inferiores, se pueden conocer los valores normales en posición unipodal y realizar comparaciones entre el miembro derecho e izquierdo o entre la pierna de apoyo dominante y secundaria, utilizada en poblaciones que realizan actividades funcionales específicas, por ejemplo jugadores de fútbol cuya pierna dominante de apoyo es el lado opuesto de la pierna dominante al patear.

Conocer los valores de normalidad en circunstancias estáticas es esencial para después conocer los valores que corresponden a grupos patalógicos con el fin de realizar comparaciones entre sí. Se puede utilizar esta herramienta para diagnosticar deficiencias en el equilibrio, obtener resultados que sirven para valorar la progresión o disminución de estas deficiencias, y para analizar las respuestas a tratamientos específicos en el trastorno de equilibrio, por ejemplo el síndrome vestibular periférico. Situaciones de uso que no se encuentran con esta revisión pero que se identifican comunmente en Colombia incluyen a la población con amputación. El control postural es un componente esencial en la adaptación a una prótesis de miembro inferior y es un enfoque dentro de los programas de fisioterapia. Se pueden utilizar las plataformas de fuerza con sensores de presión que existen en Colombia para realizar la medición en la posición bípedo, tal y como realizar comparaciones entre el lado sano y amputado con el fin de valorar el nivel de adaptción a la prótesis, los efectos de los criterios estáticos y dinámicos de la construción de la prótesis y la alineación de los componentes para dirigir la intervención fisioterapeútica y protésica hacia el usuario.
Adicionalmente, ya que no existe en Colombia un estabilómetro dinámico, se recomienda el uso de los principios de la estabilometría en el diseño de una plataforma de fuerza que mida el control postural en circunstancias dinámicas, específicamente el centro de presión plantar, las fuerzas de reación y los momentos, y permita la valoración de personas de las mismas poblaciones descritas anteriormente pero con el fin de conocer cuantitativamente el control postural y reaciones posturales cuando se somete a una situación de perturbaciones exteriores.

En el diseño de un estabilómetro dinámico se recomienda tener en cuenta las sugerencias para mejorar el rendimiento de estabilómetros dinámicos reportados en el estudio por Commissaris et al 2002 (30). 1. El tamaño pequeño de las plataformas no permite que la persona tenga el espacio para tomar un paso compensatorio como respuesta a una perturbación. 2. Las perturbaciones externas no son suficientemente grandes ni rápidas para causar inestabilidad de la persona por ejemplo en la plataforma de NeuroCom que permite $10^{\circ}$ de amplitud de rotación a una velocidad de $50^{\circ} / \mathrm{s} 3$. Las perturbaciones externas suelen ser provistas en un plano y así no son multidireccionales. 4. El alto costo que viene de los motores de torque responsables de crear las perturbaciones externas. Por estas razones en su estudio se diseñó una plataforma dinámica que simula perturbaciones multi-direccionales (hasta $19^{\circ}$ ) con una superficie suficientemente amplia para permitir la toma de un paso correctivo. (30)

En conclusión, el presente trabajo, ofrece una visión amplia sobre la aplicación de la estabilometría en los diferentes escenarios de acción del fisioterapeuta. En la clínica, en el deporte, en el trabajo, es posible medir cuantitativamente el balance postural, así como también, evaluar los resultados de su intervención para optimizar esta condición del movimiento coporal. Esta revisión sirve como guía para el desarrollo de futuras investigaciones sobre la implimentación de equipos de estabilometría como herramienta cuantitativa de medición para el área de fisioterapia y para otras áreas de la salud interesadas en valorar esta condición y relacionarla con otros factores del cuerpo humano.

\section{AGRADECIMIENTOS}

Agradecemos a los estudiantes (Juanita López, Lorena Vivas, Blanca Mora, Alejandra Daza, Giovanna Escobar, Yenny Murcia) de la Especialización en Neurorehabilitación Cohorte II de la Escuela Colombiana de Rehabilitación, por ayudar con la búsqueda de artículos científicos. 


\section{Referencia:}

\section{Las referencias a otras obras son una parte muy importante en la literatura científica; ya que estas permiten conocer más sobre los autores y mantener vivas sus voces dentro del texto.}

1. Agrawa Y, C. J. The modified Romberg Balance Test: normative data in U.S. adults. Otol Neurotol. 2011;32(8):1309-11.

2. Leddy AL, C. B. Functional gait assessment and balance evaluation system test: reliability, validity, sensitivity, and specificity for identifying individuals with Parkinson disease who fall. Phys Ther. 2011:91(1):102-13.

3. Gribble, P. H. Considerations for Normalizing Measures of the Star Excursion Balance Test. MEASUREMENT IN PHYSICAL EDUCATION AND EXERCISE SCIENCE. 2003;7 (2):89-100.

4. Shumway-Cook A, W. M. (2000). Motor Control: Theory and Practical Applications. Philadelphia:Lippincott Williams \& Wilkins.

5. Winter, D. A. Human balance and posture control during standing and walking. Gait and Posture. 1995;3(4):193-214.

6. Resourcesonbalance.com, Atención Primaria en la Red [sede Web]. Disponible en: http://www.resourcesonbalance.com/program/role/ cdp/protocols.aspx\#SOT\#SOT

7. Ageberg E, Roberts D, Holstorm E, Friden T. Balance in single-limb stance in healthy subjects reliability of testing procedure and the effect of short-duration sub-maximal cycling. BMC. Musculskelet Disord. 2003;4:14.

8. Chaudhry, H. B. Measurement of balance in computer posturography: Comparison of methodsdA brief review. Journal of Bodywork \& Movement Therapies, doi:10.1016/j.jbmt.2008.03.003.

9. Bartual J, Pérez N. El sistema vestibular y sus alteraciones. Tomo 1. Fundamentos y semiología. 1998;21-22.

10. Astaiza D, M. N. Método de evaluación propioceptiva en miembros inferiores. Buenos Aires : s.n. Efdeportes. Año 13. 2009;128. Revista Digital. Disponible en URL: HYPERLINK "http://www.efdeportes. com" http://www.efdeportes.com

11. Bruner E, Mantini S, Guerrini V, Ciccarelli A, Giombini A, Borrione P, Pigo F. Preliminary shape analysis of the outline of the baropodometric foot. Journal of Sports Medicine and Physical Fitness. 2009;49:246-52.

12. Vuillerme, N. P. How a plantar pressure-based, tongue-placed tactile biofeedback modifies postural control mechanisms during quiet standing. Exp Brain Res. 2007;181: 547-54.

13. Tekscan.com, Atención Primaria en la Red [sede Web]. Disponible en: HYPERLINK "http://www.tekscan.com/medical/pressure-sensitivemat.html” |t “_blank" http://www.tekscan.com/medical/pressuresensitive-mat.html

14. Imukai K, Koizuka I, Takahashi S. Head-tilting stabilometry in patiens with benign parosixysmal positional vertigoAuris Nasus Larynt. 2008;36:31-6.

15. Barclay - Goddard R. Stevenson T. Poluha W. Mofffan MEK. Y Taback SP. Retroalimentación en la plataforma de fuerza para el entrenamiento del equilibrio en bipedestación después de un accidente cerebro vascular. (Revisión Cochrane traducida), En: La Biblioteca Cochrane Plus. 2008;2:1-21.

16. Amti.biz, Atención primaria en la Red [sede Web]. Disponible en: HYPERLINK "http://amti.biz/"|t "_blank" http://amti.biz/

17. Lara A, Abian J, Alegre M, Jimènez L. Assessment of Power Output in jump test for applicants to a sports sciences degree. J Sports Medicine and Physical Fitness. 2006;46:1-34.

18. Nardone A, Schieppati M. Balance in Parkinson's Disease Under Static and Dynamic Conditions. Movement Disorders. 2006;21(9):151520.

19. Kistler.com, Atención Primaria en la Red [sede Web]. Disponible en: HYPERLINK "http://www.kistler.com/ch_en-ch/13_ Productfinder/6000.-.15/Product" It "_blank" http://www.kistler. com/ch_en-ch/13_Productfinder/600o.-.15/Product Finder.html

20. Gerosa M, Marsiano N, Longhi N, De Simone A, Feroni R, Verlicchi A, Zanotti B, Nicolato A. Gamma Knife surgery in vestibular schwannomas: impact on the anterior and posterior labyrinth. J Neurosurg. 2010;113: 128-35.

21. Guy-Capron.com, Atención Primaria en la Red [sede Web]. Disponible en: HYPERLINK "http://www.guy-capron.com/index.php" $\mid t$ "_blank" http://www.guy-capron.com/index.php

22. Broglio SP, Sosnoff JJ, Rosengren KS, McShane K. A Comparison of Balance Performance: Computerized Dynamic Posturography and a Random Motion Platform. Arch Phys Med Rehabil. 2009; 90:145-50.

23. NeuroCom International. Inc. (2009). Sensory Organization Test SOT: NeuroCom Protocols: NeuroCom Products. Recuperado el 20 de Septiembre de 2010, de Balance Manager Products: http://resourcesonbalance.com/neurocom/protocols/sensoryImpairment/SOT. aspx

24. Visser J, C. M. The clinical utility of posturography. Clinical Neurophysiology. 2008;119:2424-36.

25. Faculty of Rehabilitation at the University of Montpellier France. (2011). Université Montpellier. Recuperado el 20 de 08 de 2011, de en.www.univ-montp1.fr

26. Dvir Z, T. T. Instrumented stabilometer for dynamic balance studies. Med. \& Biol. Eng. \& Comput. 1982;20:19-22.

27. Giacomozzi C. Hardware performance assessment recommendations and tools for baropodometric sensor systems. Ann Super Ist Sanità. 2010;46(2):158-67.

28. Middleton J. Sinclair P. Patton R. Accuracy of center of pressure measurement using a piezoelectric force platform. Clinical Biomechanics. 1999;14:357- 60 .

29. Ageberg E, Zätterström R, Fride' T, Moritz U. Individual factors affecting stabilometry and one-leg hop test in 75 healthy subjects, aged 15-44 years. Scand J Med Sci Sports. 2001;11:47-53. 
30. Commissaris DA, Nieuwenhuijzen PH, Overeem S, de Vos A, Duysens JE, Bloem BR. Dynamic posturography using a new movable multidirectional platform driven by gravity. Journal of Neuroscience Methods. 2002;113: 73-84.

31. Collado Vázquez S. ANÁLISIS DE LA MARCHA HUMANA CON PLATAFORMAS DINAMOMÉTRICAS. INFLUENCIA DEL TRANSPORTE DE CARGA www.ucm.es/eprints/4401/o1/med3.pdf. Biblioteca Universitaria 2002.

32. Gravante G, Pomara F, Amato, F. Plantar Pressure Distribution Analysis in Normal Weight Young Women and Men With Normal and Claw Feet: A Cross-Sectional Study. Clinical Anatomy. 2005;18:245-50.

33. Johansson R, Magnusson M, Fransson PA, Karlberk M. Multi-stimulus multi-response posturography. Mathematical Biosciences. 2001;174:41-59.

34. Alfieri FM, de Jesus Guirro RR, Teodori RM. Postural stability of elderly submmitted to multisensorial physical therapy intervention. Electromyography and clinical Neurophysiology. 2010;50(2):113-9.

35. Alfieri FM, Marcelo Riberto; Lucila Silveira Gatz; Carla Paschoal corsi; Jose Augusto Fernandes; Jose maria Santarem, Linamara Rizzo Battistella. Functional mobility and balance in community dwelling elderly submitted to multisensory versus strength exercises clinical interventions in Aging 2010;5:181-5.

36. Fabris SM, Antonio Carlos Valezi AC, Fabris de S. Souza; Joel Faintuch; Ivan Cecconello; Mauro Pedroni Junior. Computerized Baropodometry In Obese Patients. Obesity Surgery. 2006;16:1574-8.

37. Gravante G, Russo G, Ridola C. Comparison of ground reaction forces between obese and control young adults during quiet standing on a baropodometric platform. Clinical Biomechanics. 2003;18:780-2.

38. Costa PB, Graves BS, Whitehurst M, Jacobs PL. The Acute Effects of Different Durations of Static Stretching on Dynamic Balance Performance. Journal of Strength and Conditioning Research. 2009;23:141-7.

39. López-Rodriguez S, Fernaández de-las-Peñas C, AlburquerqueSendiín F, Rodríguez-Blanco C, Palomeque-del-Cerro L. Inmediate effects of manipulation of the talocrural joint on stabilometry and baropodometry in patients with ankle sprain. 2007;86-192.
40. Boza R, Duarte E, Belmonte R Estudio Baropodométrico en el hemipléjico Vascular Servicio de Medicina Fisica y Rehabilitación. Hospital del Mar. Barcelona. 2007;41:3-9.

41. Ganesan M, Kumar P, Gupta A, Sathyaprabha TM. Dynamic posturography in evaluation of balance in patients of Parkinson's disease with normal pull test: Concept of a diagonal pull test. Parkinsonism and Related Disorders. 2010;16:595-9.

42. Yabe I, Matsushima M, Soma H, Basri R, Sasak H. Usefulness of the Scale for Assessment and Rating of Ataxia (SARA). Journal of the Neurological Sciences. 2008;266:164-6.

43. Yoshikawa M, Doita M, Okamoto K, Manabe M. Norihide Sha, and Masahiro Kurosaka. Impaired Postural Stability in Patients With Cervical Myelopathy. Spine. 2008;33(14):460-4.

44. Rossi C, Alberti A, Sarchielli P, Mazzotta G, Capocchi G, Faralli M, Ricci G, Molini E, Altissimi G. et al. Balance disorders in headache patients: evaluation by computerized static stabilometry. Acta Neurologica Scandanavica. 2005;111:407-13.

45. Ishizaki K, Nozomi M, Takeshima M, Yoko F, Tamami L, Kusumi M, Yasui K, Kowa H, Nakashima K. Static stabilometry in patientes with migraine and tension-type headaches. Psychiatry and Clinical Neurosciences. 2002; 56:85-90.

46. Whitney SL, Marchetti GF, Schade AL. The Relationship Between Falls History and Computerized Dynamic Posturography in Persons With Balance and Vestibular Disorders. Arch Phys Med Rehabil. 2006;87:402-7.

47. Matsuo T, Yabuki A, Hasebe K, Hirai Shira Y, Imai S. Postural stability and prism adaptation test in exotropia. Invest Ophtholom Vis Sci. 2010;51: 1-27.

48. Matsuo T, Narita A, Senda M, Hasebe S, Ohtsuki H. Body Sway Increases immidiately after strabismus surgery. Acta Med Okayama. 2006;60(1):13-24.

49. Nordahi SHG, Aasen T, Dyrkorn BM, Eidsvik S, Molvaer O. Static Stabilometry and Repeated Testing in a Normal Population. Aviation, Space, and Environmental Medicine. 2000;71:889-93.

50. Fort ARD. Diferencias en la estabilidad postural estática y dinámica según sexo y pierna dominante. Apunts med esport. 2009;162: 74-81. 